\title{
Utilisations digestives comparées des laits UHT de chèvre et de vache Effets nutritionnels de la gélification Utilisation du modèle porcin
}

\author{
C Février, J Mourot, Y Jaguelin, A Mounier, Y Lebreton
}

Station de recherches porcines, INRA, 35590 Saint-Gilles, France

\begin{abstract}
Résumé - La digestibilité des laits de chèvre et de vache a été étudiée en prenant le porc en croissance comme modèle, avec des apports en protéines et en lipides identiques entre les 2 laits. Les laits complémentaient de l'orge pour $25 \%$ de la matière sèche de la ration. Pour les nutriments majeurs (énergie, azote, fibres alimentaires) la digestibilité a été mesurée au niveau fécal chez 2 lots de 5 porcs $(35 \mathrm{~kg}$ ) pendant $10 \mathrm{j}$. Pour les acides aminés et les acides gras, 6 porcs, disposant d'une anastomose iléorectale, ont permis de mesurer la digestibilité iléale pendant au moins 5 périodes de $7 \mathrm{j}$ par traitement. L'incidence de la gélification du lait de chèvre a été étudiée en digestibilité iléale. Les 2 laits ont conduit à des croissances identiques. De même, les utilisations digestives totales de nutriments majeurs n'étaient pas différentes entre les 2 laits, mais les minéraux tendraient à être mieux absorbés avec le lait de chèvre. II en était de même en digestibilité iléale. Mais à ce niveau, la digestibilité vraie de la cystine $(-16,7$ points), de la méthionine $(-6,1$ points) et de l'isoleucine $(-6,3$ points), étaient en défaveur du lait de chèvre, alors que celle de la lysine n'était pas différente entre les 2 laits, 93,2 et $94,6 \%$ pour chèvre et vache, respectivement. Les acides gras à chaîne moyenne étaient totalement digérés dans l'intestin grèle, mais les autres tendaient à être un peu mieux absorbés à partir du lait de chèvre (C14:0 et C18:2). La gélification a eu pour effet d'inhiber l'absorption du lactose et des minéraux, de même que la dégradation des fibres alimentaires. Elle a entraîné une diminution de la digestibilité de la cystine et de l'arginine. Enfin, elle a eu peu d'effets sur les lipides, sauf sur C18:2 (-44 points). En conclusion, on peut déduire de ce modele que la valeur nutritive globale du lait de chèvre, liquide UHT, n'est pas significativement differente de celle du lait de vache UHT pour la croissance après le sevrage.
\end{abstract}

porc / lait de chèvre / lait de vache / gélification / nutrition

Summary - Comparative digestive utilization of UHT goat's and cow's milks: nutritional effects of gelation - use of a swine model. The digestive utilization of the nutrients from goat's and cow's milk was studied using the growing pig as a model. The digestibility of the main nutrients (energy, crude protein, alimentary fibre) was measured at the fecal level, and that of the amino acids and fatty acids, was determined at the ileal level, using an equal supply of crude protein and crude fat with both milks. In addition, the nutritional impact of goat's milk gelation was studied in ileal digestibility. In both experiments, the animals were fed fortified barley with goat's or cow's milk (25\% of total dry matter in the diet). Total digestibility was measured over a 10-d period in 2 groups of 5 barrows (35 $\mathrm{kg}$ liveweight) fed the 2 diets. In another group of 6 pigs, a surgically-modified ileo-rectal 
anastomosis allowed measurement of ileal digestibility over at least 5 or $7-d$ periods per treatment. With the animals used for the total digestibility, both the milks led to the same performance. These results are consistent with the fact that the total digestive utilization of the main nutrients was not significantly different between the milks. Only the minerals tended to be better absorbed from the goat's milk than from the cow's milk. This was also the case for ileal digestibility. At this level, the differences were significant: the true (conventional) digestibility of cystine ( -16.7 points), methionine ( -6.1 points) and isoleucine ( -6.3 points) was lower in goat's milk; but that of lysine did not differ between the 2 milks, ie 93.2 and $94.6 \%$ for the goat's and cow's milks respectively. The middle-chain fatty acids were completely digested in the small intestine. Although the differences between the 2 milks were not significant, the goat's milk fatty acids tended, on average, to be slightly better absorbed than the cow's milk fatty acids, especially as regards C14:0 and C18:2. The main consequence of gelation was an inhibition of the absorption of lactose and minerals and a reduction in alimentary fibre degradation, leading to a decrease in milk and diet energy content. Gelation led to an additional decrease in cystine and arginine digestibility. Finally, gelation had little effect on fats, with the exception of linoleic acid whose digestibility was reduced. In conclusion it can be inferred from this swine model that the gross nutritive value of UHT liquid goat's milk is not significantly different from that of UHT cow's milk for growth after weaning.

pig / goat's milk / cow's milk / milk gelation / nutrition

\section{INTRODUCTION}

Dans la plupart des études nutritionelles sur le lait de chèvre, l'examen de sa composition chimique sert de base à l'estimation de sa valeur nutritive, par comparaison à celle du lait de vache (Parkash et Jenness, 1968 ; Birbeck, 1985 ; Coveney et Darnton-Hill, 1985 ; Grandpierre et al, 1988). Par ailleurs, les rares études in vivo publiées portent généralement sur des mesures de croissance ou de rétention azotée (Daniels et Stearns, 1925 ; Lee et al, 1962 ; Shurpalekar et al, 1964) mais, à notre connaissance, aucune référence n'est disponible concernant la digestibilité des nutriments du lait de chèvre. Or, la première étape de l'utilisation d'un lait, indépendamment de l'équilibre entre ses nutriments, est son aptitude à être rapidement absorbé par l'intestin, et notamment par l'intestin grêle. Cette absorption dépend aussi de la nature de la ration qui l'accompagne, par exemple des sources de fibres alimentaires, mais elle dépend aussi, pour un lait donné, des traitements technologiques qui lui ont été appliqués.
Pour obtenir une approche expérimentale des potentialités d'utilisation digestive chez l'homme, le porc en croissance, animal omnivore et monogastrique, constitue un bon modèle peu onéreux.

Dans le cas de l'étude de la digestibilité comparée des nutriments du lait de chèvre et du lait de vache qui nous a été confiée, nous avons choisi de considérer globalement l'utilisation digestive des grandes classes de nutriments des 2 laits, puis de considérer plus précisément l'utilisation digestive de leurs acides aminés et de leurs acides gras dans l'intestin grêle, par la mesure de la digestibilité à la fin de l'iléon. Dans ce dernier cas, nous avons eu la possibilité de considérer les effets nutritionnels d'un accident de gélification survenu lors de la préparation d'un lot de lait de chèvre UHT.

Le principe de mesure de la valeur nutritive des laits repose sur leur aptitude à complémenter un aliment pauvre en protéines et riche en fibres alimentaires, telle que l'orge, enrichie cependant en minéraux et vitamines pour couvrir les besoins nutritionnels du porc. 


\section{MATÉRIEL ET MÉTHODES}

\section{Composition des laits et rationnement des animaux}

Les laits de vache et de chèvre, destinés à la consommation humaine post-infantile proviennent de grands mélanges et ont été traités par un procédé UHT. Pour obtenir un apport quotidien identique en lipides et en protéines entre les 2 laits, celui de chèvre était utilisé entier, tandis que celui de vache entier était coupé par du lait écrémé dans le rapport 87,5/ 12,5. Ainsi, environ $25 \%$ de la matière sèche de la ration était apportée par le lait. Compte tenu des différences de teneurs en matière sèche, le lait de vache a été fourni à raison de $2,75 \mathrm{~kg} / \mathrm{j}$ et par animal, contre $3 \mathrm{~kg}$ pour le lait de chèvre, en complément de $1 \mathrm{~kg}$ d'orge supplémentée (Orge 97,$13 ; \mathrm{CO}_{3} \mathrm{Ca} 1,0 ; \mathrm{Ca}_{2} \mathrm{HPO}_{4} 1,73 ; \mathrm{NaCl} 0,7$; oligoéléments et vitamines 0,7 ), le tout était distribué en mélange et en 2 repas équilibrés. Le même rationnement a été adopté pour les 2 expériences de digestibilité, totale et iléale. Les compositions finales moyennes des aliments et des régimes sont fournies au tableau I et les profils de compositions en acides aminés et en acides gras sont rapportés aux tableaux II et III.

\section{Mesure de la digestibilité fécale}

Cinq couples de porcs mâles castrés, croisés Large White $x$ Pietrain, ont été répartis en 2 lots selon un dispositif en blocs complets. Ils ont été logés individuellement dans des cages permettant la mesure des bilans digestifs et urinaires. Après une semaine d'adaptation aux cages puis une autre semaine d'adaptation au régime expérimental, la mesure des bilans a été effectuée pendant une période $10 \mathrm{j}$. Les porcs ont ensuite été conservés avec leurs régimes respectifs pour atteindre une durée totale d'alimentation de $28 \mathrm{j}$.

Tableau I. Composition de l'orge, des laits et des régimes expérimentaux (\% de matière sèche). Chemical composition of barley, milks and the experimental diets (\% dry matter).

\begin{tabular}{|c|c|c|c|c|c|c|c|}
\hline $\begin{array}{l}\text { Digestibilité } \\
\text { totale (T) } \\
\text { ou iléale (I) }\end{array}$ & $\begin{array}{l}\text { Lait de } \\
\text { vache }\end{array}$ & $\begin{array}{l}\text { Lait de } \\
\text { chèvre } \\
\text { normal }\end{array}$ & $\begin{array}{l}\text { Lait de } \\
\text { chèvre } \\
\text { gélifié }\end{array}$ & $\begin{array}{l}\text { Régime } 1 \\
\text { Orge } \\
\text { (en digest) } \\
\text { iléale }\end{array}$ & $\begin{array}{l}\text { Régime } 2 \\
\text { Lait de } \\
\text { vache }\end{array}$ & $\begin{array}{l}\text { Régime } 3 \\
\text { Lait de } \\
\text { chèvre }\end{array}$ & $\begin{array}{c}\text { Régime } 4 \\
\text { Lait de } \\
\text { chèvre } \\
\text { gélifié }\end{array}$ \\
\hline
\end{tabular}

\begin{tabular}{|c|c|c|c|c|c|c|c|c|}
\hline \multirow[t]{2}{*}{ Mat sèche } & $T$ & 11,83 & 10,60 & - & 88,40 & 32,25 & 30,05 & - \\
\hline & 1 & 11,63 & 10,52 & 9,67 & 88,10 & 32,02 & 29,91 & 29,27 \\
\hline \multirow[t]{2}{*}{ Mat minérale } & $\mathrm{T}$ & 5,56 & 7,37 & - & 5,54 & 5,55 & 6,02 & - \\
\hline & 1 & 5,61 & 7,24 & 7,24 & 5,27 & 5,36 & 5,79 & 5,75 \\
\hline \multirow[t]{2}{*}{ Azote } & $\mathrm{T}$ & 4,35 & 4,46 & - & 2,11 & 2,71 & 2,73 & - \\
\hline & 1 & 4,50 & 4,56 & 4,63 & 2,12 & 2,76 & 2,77 & 2,74 \\
\hline \multirow[t]{2}{*}{ Lipides } & $T$ & 24,49 & 23,33 & - & 2,45 & 8,38 & 7,98 & - \\
\hline & I & 22,79 & 23,82 & 23,84 & 2,59 & 7,90 & 8,17 & 7,91 \\
\hline \multirow[t]{2}{*}{$\mathrm{NDF}^{2}$} & $\mathrm{~T}$ & - & - & - & 23,21 & 16,97 & 17,07 & - \\
\hline & I & - & - & - & 22,97 & 16,85 & 16,91 & 17,28 \\
\hline \multirow{2}{*}{$\begin{array}{l}\text { Énergie brute } \\
\text { en cal/g }\end{array}$} & $\mathrm{T}$ & 5558 & 5481 & & 4555 & - & - & - \\
\hline & 1 & 5520 & 5461 & 5393 & 4529 & - & - & - \\
\hline
\end{tabular}

\footnotetext{
${ }^{1}$ Mélange de lait entier et de lait écrémé $(87,5 \%+12,5 \%) ;{ }^{2}$ fibres alimentaires, cf texte.
}

${ }^{1} \mathrm{~A}$ mixture of raw milk and skim milk $(87.5+12.5 \%) ;{ }^{2}$ alimentary fibres (cf text). 
Tableau II. Profil moyen de composition en acides aminés de l'orge et des laits ( $\mathrm{g} / 16 \mathrm{~g} \mathrm{~N}$ ). Mean amino acid composition of barley and milks $(g / 16 \mathrm{~g} \mathrm{~N})$.

\begin{tabular}{lrrrr}
\hline & Orge & Vache & Chèvre normal & Chèvre gélifié \\
\hline & & & & \\
Protéines (\% MS) & 12,56 & 26,69 & 26,31 & 26,12 \\
Cystine & 1,99 & 0,90 & 0,95 & 1,02 \\
A aspartique & 6,21 & 7,46 & 7,52 & 8,12 \\
Méthionine & 1,67 & 2,66 & 2,74 & 2,74 \\
Thréonine & 3,58 & 5,40 & 5,43 & 4,58 \\
Sérine & 4,62 & 5,43 & 5,59 & 5,83 \\
A glutamique & 28,66 & 24,51 & 24,74 & 25,33 \\
Glycine & 4,30 & 1,95 & 1,82 & 2,09 \\
Alanine & 4,30 & 3,37 & 3,23 & 3,43 \\
Valine & 5,33 & 7,64 & 7,79 & 7,27 \\
Isoleucine & 3,50 & 5,06 & 5,17 & 5,36 \\
Leucine & 7,56 & 10,45 & 10,57 & 10,47 \\
Tyrosine & 3,34 & 3,90 & 3,80 & 4,91 \\
Phénylalanine & 5,49 & 6,03 & 5,70 & 5,92 \\
Histidine & 2,79 & 2,81 & 2,96 & 3,07 \\
Lysine & 3,58 & 8,06 & 8,17 & 8,13 \\
Arginine & 5,01 & 2,96 & 3,04 & 3,52 \\
Proline & 12,82 & 12,22 & 12,09 & 11,07 \\
& & & & \\
\hline
\end{tabular}

Tryptophane non déterminé.

Tableau III. Profil moyen de composition en acides gras de l'orge et des laits (en $\%$ des acides gras identifiés).

Mean fatty acid composition of barley and milks (\% of identified fatty acids).

\begin{tabular}{llrrrr}
\hline & & Orge & Vache & Chèvre normal & Chèvre gélifié \\
\hline Caprylique & C8:0 & - & 3,28 & 4,15 & 5,60 \\
Caprique & C10:0 & - & 4,25 & 13,00 & 14,62 \\
Laurique & $\mathrm{C} 12: 0$ & - & 3,76 & 5,40 & 5,37 \\
Myristique & $\mathrm{C} 14: 0$ & 0,53 & 11,99 & 12,83 & 12,97 \\
Palmitique & $\mathrm{C} 16: 0$ & 32,16 & 37,44 & 34,59 & 33,97 \\
Palmitoléique & $\mathrm{C} 16: 1$ & 0,19 & 1,83 & 0,82 & 0,61 \\
Stéarique & $\mathrm{C} 18: 0$ & 2,02 & 10,23 & 6,16 & 7,09 \\
Oléique & $\mathrm{C} 18: 1$ & 18,69 & 23,21 & 17,48 & 18,66 \\
Linoléique & $\mathrm{C} 18: 2$ & 43,41 & 1,33 & 1,83 & 1,11 \\
Linolénique & $\mathrm{C} 18: 3$ & 2,88 & 2,68 & 0,35 & - \\
Arachidique & $\mathrm{C} 20: 0$ & - & - & 0,28 & - \\
& & & & & \\
Total & & 100,00 & 100,00 & 100,00 & 100,00 \\
& & & &
\end{tabular}




\section{Mesure de la digestibilité précæcale ou iléale}

Six porcs identiques aux précédents ont subi une intervention chirurgicale pour réaliser une anastomose ileo-rectale termino-terminale, avec conservation de la valvule iléo-cæcale (Laplace et al, 1985). Par cet artifice, le chyme iléal peut être récolté comme les fèces par la voie anale. Le gros intestin ainsi shunté reste en place et s'atrophie progressivement. Trois semaines après l'opération, les mesures de digestibilité iléale ont été réalisées pendant 4 semaines consécutives, entre 38 et $45 \mathrm{~kg}$ de poids vif, en alternant chaque semaine les différents régimes, lait de vache, lait de chèvre normal et lait de chèvre gélifié sur les différents porcs, selon une attribution aléatoire. De plus, pour pouvoir estimer par différence la digestibilité iléale propre aux nutriments du lait, un régime était constitué uniquement d'orge supplémentée. Comme pour l'expérience précédente, les porcs ont été placés dans des cages à bilans. Chaque semaine, la période d'adaptation à l'aliment était de $4 \mathrm{j}$ et celle de collecte de $3 \mathrm{j}$. Les coefficients d'utilisation digestive vrais des acides aminés des régimes ont été calculés en tenant compte d'une excrétion d'acides aminés endogène proportionnelle à la quantité de matière sèche ingérée (Mariscal-Landin, 1990). Les coefficients propres aux acides aminés du lait ont été calculés en admettant l'additivité des acides aminés digestibles de l'orge et du lait.

\section{Analyses chimiques}

Après détermination immédiate des matières sèches des aliments et des excrétats et lyophilisation des excrétats, les analyses suivantes ont été réalisées : matière sèche $\left(102^{\circ} \mathrm{C} \pm 2^{\circ} \mathrm{C}\right)$; cendres $\left(550^{\circ} \mathrm{C}\right)$; azote Kjeldahl ; énergie brute (calorimètre isopéribole) ; fibres alimentaires : Neutral Detergent Fiber (NDF) (Giger et al, 1987), Acid Detergent Fiber (ADF) et Acid Detergent Lignin (ADL) (Van Soest et Wine, 1967) : lipides totaux (hydrolyse et extraction à l'éther à chaud) ; extraction des acides gras à froid (Folch et al, 1957) et dosage par chromatographie phase gazeuse ; acides aminés, par chromatographie liquide, après hydrolyse et extraction.

\section{Analyses statistiques}

Les analyses statistiques ont été réalisées à l'aide des procédures ANOVA et GLM du programme d'analyse statistique SAS (1988) pour les expériences de digestibilité totale et iléale, respectivement. Pour cette dernière, on a fait apparaître les contrastes : "espèce" pour comparer les 2 régimes comportant les laits normaux, "gel" pour estimer l'effet de la gélification du lait de chèvre et "orge" pour comparer le régime orge aux 2 régimes avec le lait normal.

\section{RÉSULTATS ET DISCUSSION}

\section{Performances zootechniques}

Les apports de matière sèche et de protéines brutes ont été équivalents entre les 2 lots (tableau IV). Toutefois, compte tenu de la difficulté d'ajuster a priori l'apport de lipides, les porcs nourris au lait de vache en ont reçu en moyenne $16 \mathrm{~g}$ de plus chaque jour par rapport aux porcs nourris au lait de chèvre. Malgré cela, les vitesses de croissance et les efficacités alimentaires ont été identiques entre les 2 lots. L'apport d'orge ayant été le même avec les 2 laits, on peut en conclure que le lait de chèvre, pour un apport en nutriments majeurs équivalent à celui du lait de vache, a la même valeur nutritive globale pour la croissance.

\section{Utilisation digestive globale apparente des régimes}

Quels que soient les nutriments pris en compte dans l'utilisation digestive totale, aucune différence n'apparaît significative (tableau V). L'apport supérieur de $13 \mathrm{~g}$ de lipides $/ \mathrm{kg}$ de matière sèche dans le régime lait de vache ne se traduit pas par une augmentation correspondante de la teneur en énergie digestible de ce régime puisque 
Tableau IV. Digestibilité totale. Performances de croissance et efficacité alimentaire. Total digestibility. Performance and feed efficiency.

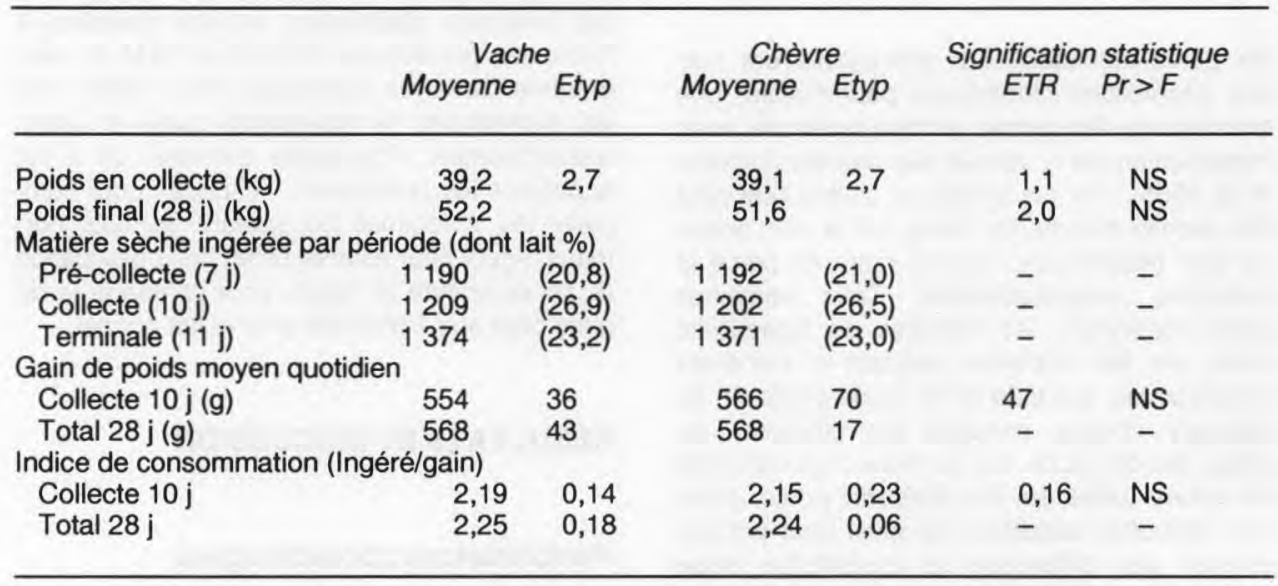

Etyp : écart type du lot ; ETR : écart type résiduel ; $\mathrm{Pr}>\mathrm{F}$ : probabilité de différence nulle entre les lots ; NS : non significatif, $\operatorname{Pr}>$ à 0,10 .

Etyp: standard deviation of the group ; ETR : residual standard deviation ; Pr > F: probability of nil difference between the groups; NS: not significant, $\mathrm{Pr}>$ at 0.10 .

Tableau V. Utilisation digestive totale apparente des régimes.

Total apparent digestive utilization of the diets.

\begin{tabular}{|c|c|c|c|c|c|c|}
\hline \multirow[t]{2}{*}{ Moyenne et écart type } & \multicolumn{2}{|c|}{ Vache } & \multicolumn{2}{|c|}{ Chèvre } & \multicolumn{2}{|c|}{ Signification statistique } \\
\hline & Moyenne & Etyp & Moyenne & Etyp & ETR & $\operatorname{Pr}>F$ \\
\hline Eau/MS ingérée & 2,58 & 0,42 & 3,33 & 0,97 & 0,66 & 0,14 \\
\hline Énergie digestible (kcal/MS) & 3702 & 39 & 3663 & 30 & 29 & 0,10 \\
\hline \multicolumn{7}{|c|}{ Coefficients d'utilisation digestive apparents (\%) } \\
\hline Matière minérale & 46,4 & 2,4 & 49,8 & 1,4 & 2,4 & 0,09 \\
\hline Énergie & 81,3 & 0,9 & 80,9 & 0,7 & 0,6 & NS \\
\hline Azote & 82,9 & 1,4 & 81,7 & 0,6 & 1,1 & NS \\
\hline Matière grasse & 70,1 & 6,0 & 69,3 & 5,7 & 4,9 & NS \\
\hline Extractif non azoté & 84,9 & 0,9 & 84,7 & 0,6 & 0,9 & NS \\
\hline Neutral detergent fiber $=$ NDF & 43,2 & 2,2 & 43,0 & 2,2 & 2,3 & NS \\
\hline Hémicellulose (NDF-ADF) & 57,8 & 1,9 & 58,2 & 2,0 & 2,1 & NS \\
\hline Cellulose (ADF-ADL) & 0,9 & 3,4 & $-1,1$ & 3,2 & 2,7 & NS \\
\hline \multicolumn{7}{|l|}{ Rétention azotée ${ }^{1}$} \\
\hline $\mathrm{N}$ retenu/j $(\mathrm{g})$ & 24,6 & 1,0 & 24,0 & 0,5 & 1,1 & NS \\
\hline $\mathrm{CRN}^{2}$ & 63,9 & 2,7 & 62,9 & 0,9 & 2,6 & NS \\
\hline CUPN ${ }^{3}$ & 53,1 & 2,2 & 51,4 & 1,1 & 2,4 & NS \\
\hline
\end{tabular}

${ }^{1} 4$ porcs dans le lot $1 ;{ }^{2}$ coefficient de rétention azoté : azote retenu/azote absorbé ; ${ }^{3}$ coefficient d'utilisation pratique de l'azote : azote retenu/azote ingéré.

${ }^{1} 4$ pigs in group $1,{ }^{2}$ nitrogen retention coefficient: retained nitrogen/absorbed nitrogen $;{ }^{3}$ nitrogen utilization coefficient: retained nitrogen/ingested nitrogen. 
la différence avec le régime "lait de chèvre" n'est que de $40 \mathrm{kcal}$, soit de l'ordre de grandeur de l'erreur de mesure.

Une différence apparaît cependant, à la limite de la signification statistique, sur la quantité d'eau ingérée, qui est supérieure avec le régime au lait de chèvre par rapport à celle mesurée avec le lait de vache. Cela peut être mis en rapport direct avec un taux de minéraux plus élevé. On note également que l'utilisation digestive des minéraux tend à être plus élevée pour le lait de chèvre que pour celui de vache $(P=$ 0,096).

Les résultats d'utilisation digestive des régimes confirment donc bien la similitude des valeurs nutritives des laits UHT de chèvre et de vache, pour des apports lipidiques et protéiques globalement équivalents.

\section{Utilisation digestive précæcale des régimes}

Les compositions moyennes des laits et des régimes propres à cette expérience sont rapportées au tableau I. Les données moyennes des niveaux alimentaires et des vitesses de croissance sont rapportées dans le tableau VI, pour les 4 lots étudiés. Les gains de poids sont donnés à titre indicatif car on observe une grande variation individuelle, surtout chez les porcs recevant l'orge seule. Cependant, pour un apport identique de protéines et de lipides entre les trois régimes au lait, les différences entre les gains de poids ne sont pas statistiquement significatives, mais l'orge seule est incapable d'assurer une croissance normale chez des porcs privés de gros intestin.

\section{Utilisation des nutriments majeurs}

Quelle que soit l'origine du lait utilisé en complément de l'orge, la digestibilité iléale de tous les éléments de la ration est améliorée par rapport à l'orge seule. Les régimes comportant le lait de vache et le lait de chèvre normal ne présentent aucune différence significative pour ce qui concerne l'utilisation digestive des nutriments majeurs (tableau VII). Les quantités d'azote retenues sont également similaires avec les 2 laits. Par rapport à ces résultats, le lait de chèvre gélifié entraîne une forte réduction de la digestibilité iléale des matières organiques et minérales, sans af-

Tableau VI. Digestibilité iléale. Performances de croissance et efficacité alimentaire. Ileal digestibility. Performance and feed efficiency.

\begin{tabular}{|c|c|c|c|c|c|c|c|c|c|}
\hline & \multirow[t]{3}{*}{ Orge } & \multirow[t]{3}{*}{ Vache } & \multirow{3}{*}{ Chèvre } & \multirow{3}{*}{$\begin{array}{l}\text { Chèvre } \\
\text { gélifié }\end{array}$} & \multicolumn{5}{|c|}{ Signification statistique } \\
\hline & & & & & \multicolumn{2}{|c|}{$\operatorname{Pr}>F$} & \multicolumn{3}{|c|}{ Contrastes } \\
\hline & & & & & ETR $F$ & Régimes & Espèce & Gel & Orge \\
\hline Nombre de périodes $(7 \mathrm{j})$ & 6 & 5 & 8 & 5 & - & - & - & - & - \\
\hline Poids moyen initial $(\mathrm{kg})$ & 39,9 & 37,4 & 36,2 & 39,9 & - & - & - & - & - \\
\hline Azote inqéré (q) & 23,4 & 32,5 & 32,9 & 32,1 & _ & _- & _- & _- & _- \\
\hline Lipides inqérés (q) & 29,6 & 93,3 & 96,7 & 92,6 & - & - & - & - & - \\
\hline Gain de poids quotidien (g) & -81 & 505 & 549 & 463 & 202 & $<0,01$ & NS & NS & $<0,01$ \\
\hline Indice de consommation & - & 2,46 & 2,74 & 3,19 & 7,50 & $0 \quad 0,02$ & NS & NS & $<0,01$ \\
\hline
\end{tabular}


Tableau VII. Utilisation digestive iléale apparente moyenne des régimes. Mean apparent ileal digestibility of the diets.

\begin{tabular}{|c|c|c|c|c|c|c|c|c|c|}
\hline & \multirow[t]{3}{*}{ Orge } & \multirow[t]{3}{*}{ Vache } & \multirow[t]{3}{*}{ Chèvre } & \multirow{3}{*}{$\begin{array}{l}\text { Chèvre } \\
\text { gélifié }\end{array}$} & \multicolumn{5}{|c|}{ Signification statistique } \\
\hline & & & & & \multicolumn{2}{|c|}{$P r>F$} & \multicolumn{3}{|c|}{ Contrastes } \\
\hline & & & & & ETR & Lot & Espèce & $\mathrm{Gel}$ & Orge \\
\hline \multicolumn{10}{|l|}{ Énergie digestible } \\
\hline (kcal/MS) & 2939 & 3509 & 3519 & 3262 & 127 & $<0,01$ & NS & $<0,01$ & $<0,01$ \\
\hline \multicolumn{10}{|c|}{ Coefficients d'utilisation digestive iléale apparents } \\
\hline Matière minérale & $-1,4$ & 13,8 & 16,2 & 8,7 & 9,9 & 0,02 & NS & NS & $<0,01$ \\
\hline Énergie & 69,7 & 76,6 & 77,0 & 72,2 & 2,8 & $<0,01$ & NS $<$ & $<0,01$ & $<0,01$ \\
\hline Azote & 71,2 & 78,2 & 78,3 & 76,5 & 2,0 & $<0,01$ & NS & NS & $<0,01$ \\
\hline Lipides & 36,0 & 70,9 & 73,1 & 69,4 & 4,3 & $<0,01$ & NS & NS & $<0,01$ \\
\hline Extractif non azoté & 59,6 & 69,6 & 69,3 & 60,6 & 4,4 & 0,03 & NS $<$ & $<0,01$ & $<0,01$ \\
\hline NDF & 34,7 & 40,7 & 41,1 & 27,1 & 9,1 & 0,07 & NS & 0,02 & NS \\
\hline Hémicellulose (NDF-AD & 44,6 & 48,6 & 49,7 & 36,9 & 8,1 & 0,08 & NS & 0,02 & NS \\
\hline Cellulose (ADF-ADL) & 4,6 & 16,5 & 15,5 & $-2,9$ & 13,9 & 0,10 & NS & 0,03 & NS \\
\hline \multicolumn{10}{|l|}{ Rétention azotée } \\
\hline $\mathrm{N}$ retenu/j (g) & 7,2 & 16,4 & 16,4 & 15,8 & 1,3 & $<0,01$ & NS & NS & $<0,01$ \\
\hline CRN ${ }^{1}$ & 42,9 & 64,7 & 63,5 & 64,4 & 4,0 & $<0,01$ & NS & NS & $<0,01$ \\
\hline CUPN ${ }^{2}$ & 30,5 & 50,6 & 49,8 & 49,3 & 3,7 & $<0,01$ & NS & NS & $<0,01$ \\
\hline
\end{tabular}

ETR, $\mathrm{Pr}>\mathrm{F}$ : $c f$ tableau IV $;{ }^{1}$ et $^{2}$ : $c f$ tableau V. ETR, $\operatorname{Pr}>\mathrm{F}$ : cf table IV; ${ }^{-1}$ and $^{2}$; cf table V.

fecter significativement celle de l'azote total ou de la matière grasse totale. C'est donc la digestibilité du lactose qui est d'abord affectée, peut être par modification de sa structure au chauffage. Sa liaison avec les protéines dans des réactions de Maillard peut aussi être évoquée pour expliquer la diminution de digestibilité de certains acides aminés (Harwalkar, 1982). La digestibilité des fibres alimentaires (selon le fractionnement de Van Soest) apportées par l'orge n'est pas modifiée par l'apport des laits UHT normaux, mais elle est significativement réduite avec le lait gélifié. Cela peut signifier que la fermentation intestinale est aussi modifiée par l'indisponibilité du lactose pour la flore intestinale. II est à noter également que la digestibilité des minéraux est réduite, de manière tou- tefois non significative en raison du fort écart type, mais on peut penser à une modification de leurs liaisons au cours du chauffage (formation de phosphate tricalcique).

Enfin, puisque la quantité d'azote retenue n'est pas significativement diminuée par la gélification, on peut penser qu'elle affecte peu en pratique la qualité des fractions protéiques.

\section{Utilisation digestive des acides aminés}

La mesure de la digestibilité vraie (conventionnelle) des acides aminés concerne les régimes constitués d'orge et de lait (tableau VIII). Des acides aminés importants, comme la thréonine, l'histidine et l'alanine, 
Tableau VIII. Utilisation digestive iléale vraie des acides aminés des régimes expérimentaux (\% des quantités ingérées).

True (conventional) ileal digestibility of the amino acids in the experimental diets (\% of intake).

\begin{tabular}{|c|c|c|c|c|c|c|c|c|c|}
\hline & \multirow[t]{3}{*}{ Orge } & \multirow[t]{3}{*}{ Vache } & \multirow[t]{3}{*}{ Chèvre } & \multirow{3}{*}{$\begin{array}{l}\text { Chèvre } \\
\text { gélifié }\end{array}$} & \multicolumn{5}{|c|}{ Signification statistique } \\
\hline & & & & & \multicolumn{2}{|c|}{$\operatorname{Pr}>F$} & \multicolumn{3}{|c|}{ Contrastes } \\
\hline & & & & & ETR & Régimes & Espèce & $\mathrm{Gel}$ & Orge \\
\hline Azote & 78,4 & 84,8 & 83,9 & 81,4 & 2,1 & $<0,01$ & NS & 0,05 & $<0,01$ \\
\hline Cystine & 77,6 & 81,0 & 76,4 & 72,2 & 3,9 & 0,02 & 0,06 & 0,07 & NS \\
\hline$A$ aspartique & 70,1 & 78,1 & 76,7 & 76,2 & 3,4 & $<0,014$ & NS & NS & $<0,01$ \\
\hline Méthionine & 77,3 & 87,9 & 84,4 & 81,9 & 3,5 & $<0,01$ & 0,10 & NS & $<0,01$ \\
\hline Thréonine & 69,1 & 76,6 & 78,7 & 76,3 & 3,8 & $<0,01$ & NS & NS & $<0,01$ \\
\hline Sérine & 75,9 & 80,0 & 77,7 & 76,1 & 3,2 & NS & NS & NS & 0,09 \\
\hline A glutamique & 87,5 & 89,0 & 88,4 & 87,6 & 1,5 & NS & NS & NS & NS \\
\hline Glycine & 72,9 & 78,5 & 75,0 & 74,1 & 3,5 & 0,10 & NS & NS & 0,05 \\
\hline Alanine & 65,3 & 71,3 & 68,6 & 66,4 & 3,7 & 0,08 & NS & NS & 0,02 \\
\hline Valine & 73,6 & 83,7 & 82,0 & 80,5 & 2,2 & $<0,01$ & NS & NS & $<0,01$ \\
\hline Isoleucine & 74,9 & 84,4 & 80,8 & 79,4 & 3,1 & $<0,01$ & 0,07 & NS & $<0,01$ \\
\hline Leucine & 77,1 & 86,1 & 84,3 & 83,0 & 1,9 & $<0,01$ & NS & NS & $<0,01$ \\
\hline Tyrosine & 75,1 & 85,8 & 83,3 & 81,0 & 2,6 & $<0,01$ & NS & NS & $<0,01$ \\
\hline Phénylalanine & 70,4 & 81,1 & 79,7 & 77,6 & 2,6 & $<0,01$ & NS & NS & $<0,01$ \\
\hline Histidine & 53,5 & 66,1 & 65,1 & 62,6 & 6,9 & 0,02 & NS & NS & $<0,01$ \\
\hline Lysine & 60,2 & 81,9 & 81,0 & 78,4 & 3,4 & $<0,01$ & NS & NS & $<0,01$ \\
\hline Arginine & 74,8 & 82,1 & 80,3 & 76,6 & 2,7 & $<0,01$ & NS & 0,026 & $<0,01$ \\
\hline Proline & 90,8 & 94,0 & 94,4 & 93,0 & 1,4 & $<0,01$ & NS & NS & $<0,01$ \\
\hline
\end{tabular}

ETR, $\operatorname{Pr}>\mathrm{F}, \mathrm{NS}$, cf tableau IV.

présentent des digestibilités faibles, quel que soit le lait. Dans le lait de chèvre normal, la cystine, la méthionine et l'isoleucine présentent une digestibilité moindre, à la limite de la signification statistique, que dans le lait de vache. Mais la digestibilité de la lysine, acide aminé le plus important, est identique dans les 2 régimes : 81,9 et $81,0 \%$. La gélification du lait de chèvre tend à réduire l'utilisation digestive des acides aminés, mais la différence ne devient significative que pour la cystine et l'arginine.

En tenant compte des apports de l'orge en acides aminés digestibles, le résultat du calcul de la digestibilité propre des acides aminés des laits est rapporté dans le tableau IX. Ce calcul accentue les différences d'utilisation digestive des acides aminés entre les laits, particulièrement pour le lait gélifié.

Ce résultat concernant l'incidence des traitements technologiques sur la digestibilité des acides aminés pourrait expliquer en partie les résultats dans lesquels le lait de chèvre est présenté comme inférieur à celui de vache pour la croissance ou la rétention azotée (Daniels et Stearns, 1925 ; Lee et al, 1962), dans la mesure où ces traitements ne sont pas pris en compte. Or on sait que le lait de chèvre est plus instable à la chaleur que celui de vache. 
Tableau IX. Utilisation digestive vraie des acides aminés des laits (calcul par différence).

True ileal digestibility of the amino acids in the milks (calculated by difference).

\begin{tabular}{llll}
\hline & Vache & Chèvre & $\begin{array}{c}\text { Chèvre } \\
\text { gélifié }\end{array}$ \\
& & & \\
\hline & & & \\
Azote & 93,9 & 91,6 & 86,0 \\
Cystine & 89,6 & 72,9 & 55,5 \\
A aspartique & 86,2 & 84,0 & 83,6 \\
Méthionine & 96,5 & 90,4 & 85,9 \\
Thréonine & 84,3 & 87,0 & 83,3 \\
Sérine & 84,4 & 79,7 & 76,3 \\
A glutamique & 91,3 & 89,7 & 87,9 \\
Glycine & 93,5 & 81,1 & 78,2 \\
Alanine & 81,1 & 74,0 & 68,5 \\
Valine & 93,4 & 89,7 & 87,4 \\
Isoleucine & 92,5 & 86,2 & 83,7 \\
Leucine & 94,7 & 91,2 & 89,1 \\
Tyrosine & 95,5 & 92,6 & 88,5 \\
Phénylalanine & 94,2 & 90,8 & 87,8 \\
Histidine & 81,2 & 80,3 & 75,1 \\
Lysine & 94,6 & 93,2 & 90,0 \\
Arginine & 95,8 & 92,7 & 80,9 \\
Proline & 98,8 & 99,4 & 96,5 \\
\hline
\end{tabular}

\section{Utilisation digestive des acides gras}

Pour les acides gras, il ne peut être calculé de digestibilité vraie puisque I'on ne peut actuellement estimer la production endogène. Les acides gras à chaîne moyenne, provenant exclusivement du lait, et en quantité plus importante dans le lait de chèvre sont totalement digérés dans l'intestin grèle (tableau X). À l'inverse de ce que nous avons observé pour les acides aminés, les acides gras du lait de chèvre tendent à être un peu mieux digérés que ceux du lait de vache, mais aucune différence n'est significative entre les 2 laits normaux. La gélification a également peu d'influence sur la digestibilité des acides gras, sauf pour celle de l'acide linoléique qui est diminuée. Si l'on admet que la digestibilité des lipides de l'orge n'est pas modifiée par la présence du lait, ce qu'il est difficile de vérifier dans nos conditions expérimentales, le calcul par différence à la digestibilité apparente totale des régimes permet de proposer les valeurs de digestibilité apparente propre aux acides gras des laits (tableau XI). Globalement,

Tableau $\mathbf{X}$. Utilisation digestive apparente des acides gras des régimes expérimentaux $(\%$ de l'ingéré).

Apparent ileal digestibility of the fatty acids in the experimental diets (\% of intake).

\begin{tabular}{|c|c|c|c|c|c|c|c|c|c|}
\hline & \multirow[t]{3}{*}{ Orge } & \multirow[t]{3}{*}{ Vache } & \multirow{3}{*}{ Chèvre } & \multirow{3}{*}{$\begin{array}{l}\text { Chèvre } \\
\text { gélifié }\end{array}$} & \multicolumn{5}{|c|}{ Signification statistique } \\
\hline & & & & & \multicolumn{2}{|c|}{$\operatorname{Pr}>F$} & \multicolumn{3}{|c|}{ Contrastes } \\
\hline & & & & & ETR & Régimes & Espèce & Gel & Orge \\
\hline Caprylique & - & 100 & 100 & 100 & - & - & - & - & - \\
\hline Caprique & - & 99,5 & 99,6 & 99,5 & 0,3 & $<0,01$ & NS & NS & - \\
\hline Laurique & - & 97,3 & 97,6 & 98,4 & 1,1 & $<0,01$ & NS & NS & - \\
\hline Myristique & 64,4 & 81,1 & 87,1 & 87,8 & 10,1 & $<0,01$ & NS & NS & $<0,01$ \\
\hline Palmitique & 79,1 & 74,5 & 78,0 & 77,6 & 6,1 & NS & NS & NS & NS \\
\hline Palmitoléique & 65,2 & 98,9 & 98,8 & 98,2 & 6,2 & $<0,01$ & NS & NS & $<0,01$ \\
\hline Stéarique & 63,6 & 71,6 & 76,0 & 75,6 & 7,8 & 0,04 & NS & NS & 0,02 \\
\hline Oléique & 81,9 & 95,1 & 95,1 & 94,4 & 3,4 & NS & NS & NS & $<0,01$ \\
\hline Linoléique & 93,6 & 90,5 & 92,9 & 89,4 & 2,7 & NS & NS & 0,04 & NS \\
\hline Linolénique & 97,4 & 99,3 & 98,0 & 97,6 & 2,5 & NS & NS & NS & NS \\
\hline Arachidonique & - & - & 89,1 & - & 7,6 & & & & \\
\hline Moyenne des AG & 85,8 & 84,2 & 88,1 & 87,5 & 0,08 & NS & NS & NS & NS \\
\hline
\end{tabular}


Tableau XI. Utilisation digestive apparente des acides gras des laits (calcul par différence). Apparent ileal digestibility of the fatty acids in the milks (calculated by difference).

\section{Vache Chèvre Chèvre gélifié}

\begin{tabular}{llrrr} 
Caprylique & C8:0 & 100,0 & 100,0 & 100,0 \\
Caprique & C10:0 & 99,5 & 99,6 & 99,5 \\
Laurique & C12:0 & 97,3 & 97,6 & 98,4 \\
Myristique & C14:0 & 81,3 & 87,3 & 88,1 \\
Palmitique & C16:0 & 73,5 & 77,8 & 77,2 \\
Palmitoléique $16: 1$ & 99,8 & 100,8 & 101,1 \\
Stéarique & C18:0 & 72,0 & 77,0 & 76,5 \\
Oléique & C18:1 & 97,9 & 98,6 & 97,8 \\
Linoléique & C18:2 & 64,0 & 88,9 & 45,1 \\
Linolénique & C18:3 & 99,8 & 99,2 & - \\
Total des acides gras & 83,8 & 88,7 & 88,0 \\
\hline
\end{tabular}

on retrouve les relations classiques entre la digestibilité et la longueur et/ou le degré de saturation des acides gras.

\section{CONCLUSIONS}

En conclusion, le remplacement du lait de vache par le lait de chèvre dans un régime après le sevrage ne modifie pas les caractéristiques générales de la croissance ni de la digestibilité des nutriments majeurs de la ration. Seule la cystine, dans nos conditions expérimentales, tend à être moins digestible dans le lait de chèvre que dans celui de vache. En revanche, les acides gras du lait de chèvre auraient tendance à être légèrement mieux digérés que ceux du lait de vache, sans atteindre le seuil de signification statistique, dans nos conditions expérimentales. Un autre aspect de l'étude, non rapporté ici, montre que cet équilibre différent des acides gras digestibles entre les 2 laits peut être associé à une diminution des teneurs en triglycérides et du cholestérol hépatique ou cir- culant à la fin de la période d'alimentation de 28 j (Mourot et al, 1992).

Un autre enseignement de ce travail concerne les effets nutritionnels de la gélification du lait. Celle-ci diminue fortement la valeur énergétique du lait, mais elle a aussi une action négative sur la digestibilité des acides aminés. Dans le cas présent, cela peut être mis en relation avec un chauffage prolongé (ou excessif) du lait avant la procédure UHT.

\section{RÉFÉRENCES}

Birbeck JA (1985) Goat milk in infant nutrition. N Z Med J 97, 413-414

Coveney J, Darnton-Hill I (1985) Goat's milk and infant feeding. Med J Aust 143, 508-510

Daniels AL, Stearns G (1925) The nitrogen and mineral balances in infant receiving cow' and goat's milk. Am J Dis Child 30, 359-366

Folch H, Less M, Sloane-Stanley GH (1957) A simple method for the isolation and purification of total lipids from animal tissues. $J$ Biol Chem 226, 497-509

Giger $S$, Thivend $P$, Sauvant D, Dorleans $M$, Journaix $P$ (1987) Étude de l'influence préalable de différentes enzymes amylolytiques sur la teneur en résidu NDF d'aliments du bétail. Ann Zootech 36, 39-48

Grandpierre C, Ghisolfi J, Thouvenot JP (1988) Étude biochimique du lait de chèvre. Cah Nutr Diét 23, 367-374

Harwalkar VR (1982) Age gelation of sterilized milks. In: Developments in dairy chemistry 1 Proteins (Fox PF, ed) Applied Science Publ, London, 229-260

Laplace JP, Darcy-Vrillon B, Picard M (1985) Evaluation de la disponibilité des acides aminés - choix raisonné d'une méthode. Journées Rech Porcine en France 17, 353-370

Lee M, Mehta R, Lucia SP (1962) Amino acids composition and nutritive value of goat milk casein. Proc Soc Exp Biol Med 10, 110-115

Mariscal-Landin G, Lebreton Y, Seve B (1990) Mesure, chez le porc, de l'excrétion d'acides aminés d'origine endogène, au niveau iléal, selon 2 méthodes. Journées Rech Porcine en France 22, 215-222 
Mourot J, Février C, Jaguelin Y, Mounier A (1992) Valeurs nutritives comparées du lait de vache et de chèvre chez le porc. Cah Nutr Diét 27, 351 (Abstract)

Parkash S, Jenness R (1968) The composition and characteristics of goat's milk: a review. Dairy Sci Abstr 30, 67-87

SAS (1988) SAS User's Guide: Statistics SAS Inst, Inc, Cary, NC
Shurpalekar SR, Chandrasekhara MR, Swaminathan $M$, Sreenivasan $A$, Subrahmanyan V (1964) Studies on the amino acid composition and nutritive value of the proteins of goat's milk. J Nutr Diet 1, 25-27

Van Soest PJ, Wine RH (1967) Use of detergents in the analysis of fibrous feeds. V. Determination of plant cell-wall constituents. J Assoc Off Anal Chem 50, 50-55 